\title{
CARACTERÍSTICAS FITOSSOCIOLÓGICAS DA ARBORIZAÇÃO VIÁRIA DE BONITO-MS
}

\author{
PHYTOSOCIOLOGICAL CHARACTERISTICS OF THE STREET TREES OF BONITO- \\ $M S$
}

Kendra Zamproni ${ }^{1}$, Daniela Biondi² ${ }^{2}$ Tamara Ribeiro Botelho de Carvalho Maria ${ }^{3}$, Rogério Bobrowski ${ }^{4}$

\section{RESUMO}

Diagnosis through the street trees' assessment is fundamental to the planning and maintenance of this asset, in order to maximize the benefits provided by the urban trees. Phytosociological studies allow an improvement of the data analysis obtained from the inventory, however they are still not used often in research on this subject. Within this context, the objective of this study was to analyze the phytosociological characteristics of the street trees of Bonito-MS, from the data obtained through a previous urban forest inventory. For this, the following factors were analyzed: frequency, density, dominance (expressed by the crown area in replace of the $\mathrm{DBH}$ ) and importance value, calculated by the traditional method and by the Species Performance Index (IPE). Within the results, it is possible to verify that the importance value highlighted species which presented larger crown area even to the detriment of those that presented higher absolute frequency. The species with the highest frequency, density and dominance values was Licania tomentosa, and, despite the homogeneity of its planting in the municipality, this species presented high IPE value. The use of these phytosociological parameters allows a better evaluation of the adaptation and performance of the species in the municipality and assists in the decision-making of new plantings.

Palavras-chave: Inventário; Índice de Performance; Planejamento.

\begin{abstract}
Diagnosis through the street trees' assessment is fundamental to the planning and maintenance of this asset, in order to maximize the benefits provided by the urban trees. Phytosociological studies allow an improvement of the data analysis obtained from the inventory, however they are still not used often in research on this subject. Within this context, the objective of this study was to analyze the phytosociological characteristics of the street trees of Bonito-MS, from the data obtained through a previous urban forest inventory. For this, the following factors were analyzed: frequency, density, dominance (expressed by the crown area in replace of the $\mathrm{DBH}$ ) and importance value, calculated by the traditional method and by the Species Performance Index (IPE). From the founded results, it is possible to verify that the importance value highlighted species that presented larger crown area even to the detriment of those that presented higher absolute frequency. The species with the highest frequency, density and dominance values was Licania tomentosa, but, despite the homogeneity of its planting in the municipality, this species presented high IPE value. The use of these phytosociological parameters allows a better evaluation of the adaptation and performance of the species in the municipality and assists decisions-making about new planting.
\end{abstract}

Keywords: Inventory; Performance Index; Planning.

Recebido em 05.08.2019 e aceito em 22.10.2019

1 Engenheira Florestal. MSc. Universidade Federal do Paraná. Curitiba/PR. Email: kendra.zam@gmail.com

2 Engenheira Florestal. Prof ${ }^{\underline{a}} \mathrm{Dr}^{\mathrm{a}}$ da Universidade Federal do Paraná. Curitiba/PR. Email: dbiondi@ufpr.br

3 Engenheira Florestal. MSc. Doutoranda na Universidade Federal do Paraná. Curitiba/PR. Email: trbotelhomaria@gmail.com

4 Engenheiro Florestal. Prof. Dr. da Universidade Estadual do Centro-Oeste. Irati/PR. Email: bobrowski_roger@yahoo.com.br 


\section{INTRODUÇÃO}

Os inventários florestais urbanos são fontes de dados importantes para pesquisadores que estudam os serviços ambientais, econômicos e sociais fornecidos pelas árvores urbanas e para os administradores desse patrimônio (ARAÚJO; ARAÚJO, 2011; NIELSEN; ÖSTBERG; DELSHAMMAR, 2014).

Bobrowski (2014) afirma que apesar da diversidade de informações obtidas com o inventário da arborização viária, costuma-se analisá-las parcialmente, com técnicas simples, de modo a atender objetivos específicos. Em função disso, sugere que é necessário o aprimoramento da análise de dados para melhorar a oferta de resultados úteis ao órgão público responsável pela arborização viária e para valorizar o componente arbóreo do município. Dentre outras formas de análise, destaca-se a análise fitossociológica como ferramenta importante para o planejamento.

Para Restrepo (2007), a gestão da arborização viária deve transcender o enfoque botânico e florístico, representado na valoração e recomendação de indivíduos ou espécies, para uma perspectiva ecológica-estrutural na análise e manejo da vegetação urbana, considerando as árvores como parte de uma comunidade vegetal (em áreas ou corredores).

Sendo assim, a fitossociologia é uma ciência voltada ao estudo das comunidades vegetais (GIEHL; BUDKE, 2011) e tem por objetivo a compreensão das causas e efeitos da interação entre plantas em um ambiente, da estrutura dos agrupamentos vegetais e dos processos que influenciam na continuidade ou mudança de uma comunidade vegetal ao longo do tempo (MORO; MARTINS, 2011). Estudos fitossociológicos relativos à arborização viária são pouco difundidos, tanto a determinação quanto o uso de parâmetros fitossociológicos ainda são primários nesta área de atuação (LIMA NETO, 2014).

Atualmente, os inventários de arborização viária realizados no Brasil tendem a utilizar somente a frequência ou densidade como fator descritivo da ordenação das espécies avaliadas, não fazendo uso de todos os parâmetros constituintes da análise fitossociológica (frequência, densidade, dominância e valor de importância) que são geralmente empregados no estudo de áreas naturais (BOBROWSKI, 2014).

Estudos fitossociológicos desenvolvidos no meio urbano são instrumentos para a gestão da arborização, estabelecendo informações importantes sob os aspectos ecológico, estrutural e silvicultural (LIMA NETO, 2014).

Dentro deste contexto, o objetivo deste trabalho foi analisar as características fitossociológicas da arborização viária de Bonito-MS, a partir dos dados obtidos por meio do inventário florestal urbano. 


\section{MATERIAL E MÉTODOS}

O município de Bonito localiza-se a sudoeste do estado do Mato Grosso do Sul, na região centro-oeste do Brasil (Figura 1), e faz parte da unidade geomorfológica denominada Serra da Bodoquena. O núcleo urbano do município com as coordenadas $21^{\circ} 07^{\prime} 16^{\prime \prime} \mathrm{S}$ e 56²8'55" O, está a 250 km da capital do estado, Campo Grande (FEHLAUER et al., 2010).

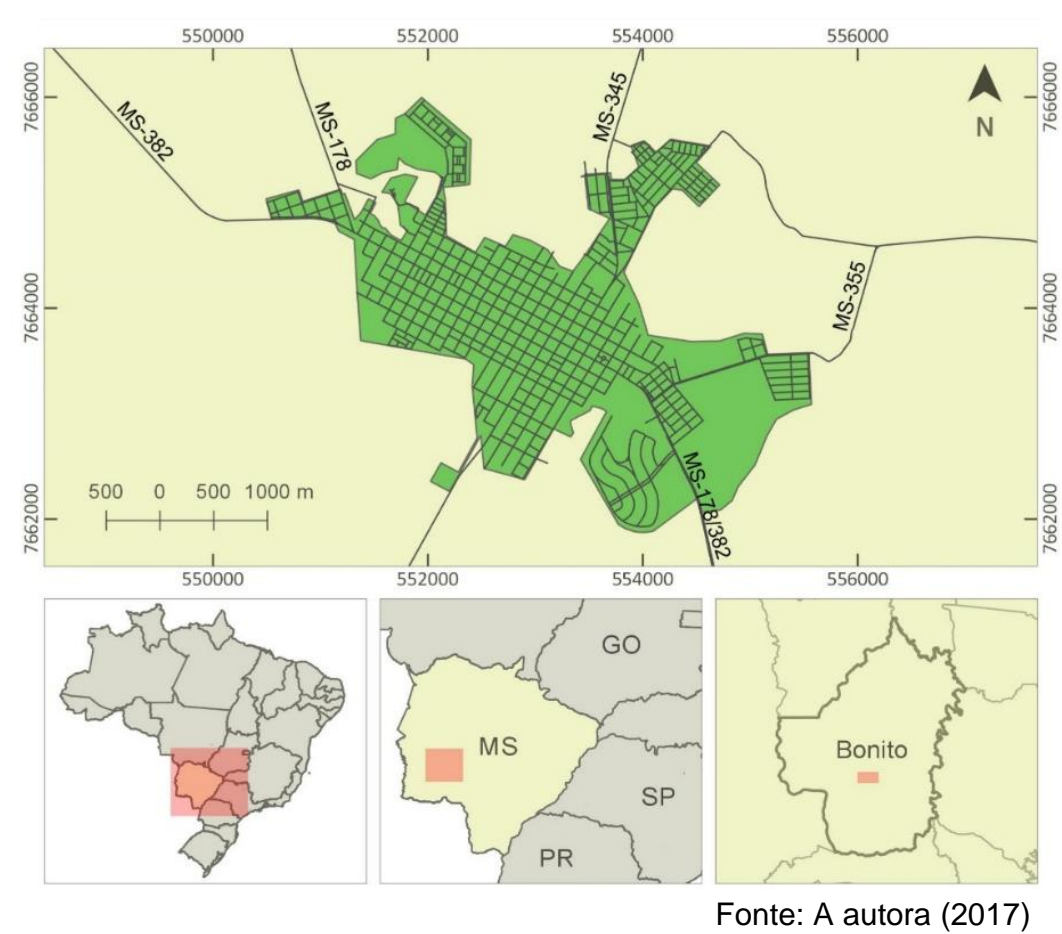

Figura 1. Localização geográfica do município de Bonito-MS, com destaque para a área urbana Figure 1. Geographic location of Bonito-MS, highlighting the urban area of the municipality

O clima da região é o Aw (tropical úmido), segundo a classificação de Köppen, caracterizado por estação seca acentuada entre os meses de junho e setembro e precipitações concentradas entre novembro e janeiro. A temperatura média anual é de $23,1^{\circ} \mathrm{C}$ e a precipitação varia entre 750 e 1800 mm ao ano (GONÇALVES; PEREIRA; DA COSTA, 2006).

Para a caracterização fitossociológica da arborização viária foram analisados os seguintes parâmetros: frequência (absoluta e relativa), densidade (absoluta e relativa), dominância (absoluta e relativa) e valor de importância, a partir dos dados obtidos em inventário florestal urbano realizado no município por Zamproni et al. (2018).

Os parâmetros fitossociológicos foram calculados de acordo com as equações descritas por Moro e Martins (2011) e adaptadas por Bobrowski (2015), como seguem: 
a) Frequência

$$
\begin{gathered}
F A=100 \frac{P e}{P t} \\
F R=100 \frac{F A}{\sum F A}
\end{gathered}
$$

Em que:

$F A$ = frequência absoluta;

$\mathrm{FR}$ = frequência relativa;

$\mathrm{Pe}=$ número total de unidades amostrais em que foi observado a espécie "e";

$\mathrm{Pt}=$ número total de unidades amostrais utilizadas no trabalho;

$\Sigma F A=$ somatório da frequência absoluta de todas as espécies amostradas.

b) Densidade

$$
\begin{gathered}
D A=\frac{n e}{A} \\
D R=\frac{D A}{\Sigma \mathrm{DA}}
\end{gathered}
$$

Em que:

$\mathrm{DA}=$ densidade absoluta;

$\mathrm{DR}=$ densidade relativa;

ne = número total de indivíduos da espécie "e";

$A=$ área total amostrada (hectares);

$\Sigma \mathrm{DA}=$ somatório da densidade absoluta de todas as espécies amostradas.

c) Dominância

$$
\begin{gathered}
D o A=\frac{A C T e}{\mathrm{~A}} \\
D o R=100 \frac{A C T e}{\Sigma \mathrm{ACT}}
\end{gathered}
$$

Em que:

DoA = dominância absoluta;

DoR = dominância relativa;

$\mathrm{A}=$ área total amostrada (hectares);

$\mathrm{ACTe}$ = área de copa total amostrada da espécie "e" $\left(\mathrm{m}^{2}\right)$;

$\Sigma A C T=$ somatório da área de copa total amostrada.

d) Valor de Importância

$$
\text { Vle }=\mathrm{FRe}+\mathrm{DRe}+\mathrm{DoRe}
$$

Em que: 
Vle = valor de importância de espécie "e";

$\mathrm{DRe}=$ densidade relativa da espécie "e";

$\mathrm{FRe}$ = frequência relativa da espécie "e";

$\mathrm{DoRe}=$ dominância relativa da espécie "e".

Para o cálculo da dominância foi utilizada a área de copa em substituição ao DAP, comumente utilizado em estudos de ecologia florestal, pois esta variável é a principal fonte de oferta de benefícios estéticos e ambientais para o meio urbano (BOBROWSKI; BIONDI, 2012).

Calculou-se ainda o Índice de Performance da Espécie (IPE), conforme Bobrowski (2014), que expressa a proporção de árvores de cada espécie que estão em condições boas e satisfatórias em relação ao total das árvores amostradas. A classificação das árvores foi definida com base em Milano (1984), como:

a) árvore boa - não apresenta sinais de pragas, doenças ou injúrias mecânicas, apresenta a forma característica da espécie;

b) árvore satisfatória - apresenta condição física e vigor medianos, aquela que sofreu podas pesadas, mas que conseguiu se reestabelecer satisfatoriamente ou que necessita reparo de danos físicos ou controle de pragas ou doenças;

c) árvore ruim - apresenta muitos danos físicos, ataque de pragas ou doenças, tortuosidade;

d) árvore muito ruim - apresentam danos físicos severos, que requerem muito trabalho de recuperação, morte iminente;

e) árvore morta.

O índice de performance da espécie (IPE) foi determinado pela equação:

$$
I P E=\frac{\left(\frac{n B+n S}{n e}\right)}{\left(\frac{N B+N S}{N}\right)} * 100
$$

Em que:

nB = número de indivíduos da espécie "e" classificados como Bons;

nS = número de indivíduos da espécie "e" classificados como Satisfatórios;

ne = número total de indivíduos da espécie "e";

$\mathrm{NB}$ = número total de indivíduos classificados como Bons;

$\mathrm{NS}$ = número total de indivíduos classificados como Satisfatórios;

$\mathrm{N}=$ número total de indivíduos.

Bobrowski (2014) explica que valores de IPE maiores que 1,0 demonstram uma boa performance da espécie na arborização viária, enquanto espécies com valores menores que 1,0 
apresentam problemas em relação à adaptabilidade, sejam estes devido a fatores ambientais e ecofisiológicos ou por práticas de manejo ou intervenções feitas pelo homem.

Com o índice de performance das espécies, recalculou-se o valor de importância (VI) por espécie, substituindo a densidade relativa (DRe) pelo IPE relativo.

\section{RESULTADOS E DISCUSSÃO}

No inventário da arborização viária realizado em Bonito-MS foram amostrados 947 indivíduos arbóreos e não arbóreos, distribuídos em 61 espécies pertencentes a 23 famílias botânicas (ZAMPRONI et al., 2018). A Tabela 1 apresenta as 15 espécies de maior valor de importância na arborização viária de Bonito-MS, de acordo com a análise fitossociológica realizada, baseada nos parâmetros: densidade, frequência e dominância, expressa pela área de copa.

Tabela 1. As 15 espécies de maior valor de importância na arborização viária de Bonito-MS e respectivos parâmetros fitossociológicos

Table 1. The 15 species with higher importance value in the afforestation of Bonito-MS and its respective phytosociological parameters

\begin{tabular}{|c|c|c|c|c|c|c|c|}
\hline \multicolumn{8}{|c|}{ ANÁLISE FITOSSOCIOLÓGICA } \\
\hline ESPÉCIE & VI & DA & DR & FA & $\mathbf{F R}$ & DoA & DoR \\
\hline Licania tomentosa (Benth.) Fritsch & 172,03 & 134,29 & 64,02 & 100,00 & 9,83 & 461114,69 & 98,1850 \\
\hline Caesalpinia pluviosa DC. & 11,17 & 8,66 & 4,13 & 64,71 & 6,36 & 3196,20 & 0,6806 \\
\hline Bauhinia sp & 7,93 & 5,47 & 2,61 & 52,94 & 5,20 & 569,61 & 0,1213 \\
\hline Murraya exótica L. & 7,51 & 4,79 & 2,28 & 52,94 & 5,20 & 137,40 & 0,0293 \\
\hline Ipê (não identificado) & 6,87 & 6,16 & 2,93 & 35,29 & 3,47 & 2190,44 & 0,4664 \\
\hline Pachira aquática Aubl. & 6,67 & 5,24 & 2,50 & 41,18 & 4,05 & 559,56 & 0,1191 \\
\hline Mangifera indica L. & 6,42 & 3,65 & 1,74 & 47,06 & 4,62 & 282,23 & 0,0601 \\
\hline Tabebuia avellanedae Lorentz ex Griseb. & 6,22 & 3,19 & 1,52 & 47,06 & 4,62 & 335,74 & 0,0715 \\
\hline Tabebuia roseoalba (Ridl.) Sandwith & 4,98 & 4,33 & 2,07 & 29,41 & 2,89 & 137,40 & 0,0293 \\
\hline Schinus molle L. & 3,78 & 1,82 & 0,87 & 29,41 & 2,89 & 85,88 & 0,0183 \\
\hline Delonix regia (bojer ex hook.) raf & 3,46 & 1,14 & 0,54 & 29,41 & 2,89 & 133,46 & 0,0284 \\
\hline Lagerstroemia indica L. & 3,17 & 2,96 & 1,41 & 17,65 & 1,73 & 96,38 & 0,0205 \\
\hline Malpighia emarginata DC. & 3,08 & 1,60 & 0,76 & 23,53 & 2,31 & 22,06 & 0,0047 \\
\hline Psidium guajava L. & 2,86 & 1,14 & 0,54 & 23,53 & 2,31 & 22,46 & 0,0048 \\
\hline Ficus sp. & 2,53 & 1,60 & 0,76 & 17,65 & 1,73 & 176,55 & 0,0376 \\
\hline
\end{tabular}

LEGENDA: $\mathrm{VI}=$ valor de importância; $\mathrm{DA}=$ densidade absoluta; $\mathrm{DR}=$ densidade relativa; $\mathrm{FA}=$ frequência absoluta; $F R$ = frequência relativa; $D o A$ = dominância absoluta; DoR = dominância relativa.

A partir dos resultados do inventário realizado, verifica-se o predomínio da espécie Licania tomentosa (Benth.) Fritsch., popularmente conhecida como oiti, com 589 indivíduos, que 
representam 63,27\% da arborização viária de Bonito-MS (ZAMPRONI et al., 2018). Portanto, tal valor de frequência da espécie nas ruas do município justifica os valores elevados dos parâmetros fitossociológicos analisados.

A predominância de $L$. tomentosa também foi verificada na arborização de ruas de outras cidades como Jerônimo Monteiro-ES (SILVA; CARDOSO; RAPHAEL, 2012), Cajuri-MG (SILVA; GONÇALVES, 2012) e Israelândia-GO (LARA; ALVES; CARNEIRO, 2014). Isto pode ser um reflexo da adaptabilidade da espécie às condições adversas do ecossistema urbano, como demonstra o bom índice de performance desta espécie neste estudo. A preferência por esta espécie na composição da arborização viária pode ser justificada por sua copa densa e perenifólia que proporciona excelente sombreamento e por ter um sistema radicial profundo. Além disso, adapta-se a regiões de clima quente, como o norte do país e regiões litorâneas (LORENZI, 2008). Entretanto, Ferreira, Gasparotto e Lima (2001) alertam que o plantio em larga escala de L. tomentosa (oiti) deve ser evitado em função do aparecimento de ferrugem causada por Phakopsora tomentosae, que ocasiona a morte da árvore, conforme encontrado pelos autores em Manaus-AM.

Santos, José e Souza (2013), em uma análise fitossociológica de espécies arbóreas das praças centrais do município de Gurupi-TO, também encontraram maior representatividade, expressa pela densidade relativa, das espécies $L$. tomentosa e $C$. pluviosa, que juntas representaram mais de $56 \%$ de todos os indivíduos levantados na área.

Conforme Zamproni et al. (2018), durante a coleta de dados do referido inventário, não foi possível identificar algumas árvores conhecidas como "ipês", pois estas não estavam no período de floração, o que inviabilizou a taxonomia a nível de gênero e espécie

Para refinar a análise dos dados, realizou-se uma segunda análise fitossociológica excluindo-se os indivíduos de L. tomentosa (oiti), tendo em vista a concentração desta espécie na arborização de ruas do município. Os resultados são apresentados na Tabela 2.

Quando comparadas as análises fitossociológicas com e sem os indivíduos de $L$. tomentosa verifica-se uma mudança no ranking das espécies. As espécies que ganharam posições, desconsiderando a mudança decorrente da saída do oiti do primeiro lugar, foram: ipê (não identificado) de $5^{\circ}$ para $2^{\circ}$ lugar, P. aquatica (embiratanha) de 6ำ para 4ํㅜ T. avellanedae (ipê-rosa) de $8^{\circ}$ para 6은 L. indica (extremosa), de $12^{\circ}$ para $9^{\circ}$ e Ficus sp. (fícus) de $15^{\circ}$ para $12^{\circ}$ lugar. Além disso, a espécie T. catappa (sombrero) passou a compor o ranking, ocupando a 14ํㅡㄴ posição. M. exotica (murta) e $P$. guajava (goiabeira) passaram a ocupar posições inferiores no ranking. 
Tabela 2. As 15 espécies de maior valor de importância na arborização viária de Bonito-MS, excluindo os indivíduos de Licania tomentosa, e respectivos parâmetros fitossociológicos

Table 2. The 15 species with higher importance value in the afforestation of Bonito-MS, excluding the trees of Licania tomentosa, and its respective phytosociological parameters

\begin{tabular}{lrrrrrrr}
\hline \multicolumn{7}{c}{ ANÁLISE FITOSSOCIOLÓGICA } & \\
\hline ESPÉCIE & VI & DA & DR & FA & FR & DoA & DoR \\
\hline Caesalpinia pluviosa DC. & 56,03 & 8,66 & 11,48 & 64,71 & 7,05 & 3196,20 & 37,4976 \\
Ipê (não identificado) & 37,70 & 6,16 & 8,16 & 35,29 & 3,85 & 2190,44 & 25,6981 \\
Bauhinia sp. & 19,70 & 5,47 & 7,25 & 52,94 & 5,77 & 569,61 & 6,6827 \\
Pachira aquática Aubl. & 18,00 & 5,24 & 6,95 & 41,18 & 4,49 & 559,56 & 6,5647 \\
Tabebuia avellanedae Lorentz ex Griseb. & 13,30 & 3,19 & 4,23 & 47,06 & 5,13 & 335,74 & 3,9388 \\
Mangifera indica L. & 13,27 & 3,65 & 4,83 & 47,06 & 5,13 & 282,23 & 3,3111 \\
Tabebuia roseoalba (Ridl.) Sandwith & 10,56 & 4,33 & 5,74 & 29,41 & 3,21 & 137,40 & 1,6119 \\
Lagerstroemia indica (L.) Pers. & 6,98 & 2,96 & 3,93 & 17,65 & 1,92 & 96,38 & 1,1308 \\
Schinus molle L. & 6,63 & 1,82 & 2,42 & 29,41 & 3,21 & 85,88 & 1,0076 \\
Delonix regia (bojer ex hook.) raf & 6,28 & 1,14 & 1,51 & 29,41 & 3,21 & 133,46 & 1,5657 \\
Ficus sp. & 6,11 & 1,60 & 2,12 & 17,65 & 1,92 & 176,55 & 2,0713 \\
Malpighia emarginata DC. & 4,94 & 1,60 & 2,12 & 23,53 & 2,56 & 22,06 & 0,2588 \\
Terminalia catappa L. & 4,64 & 0,91 & 1,21 & 17,65 & 1,92 & 128,61 & 1,5089 \\
Psidium guajava L. & 4,34 & 1,14 & 1,51 & 23,53 & 2,56 & 22,46 & 0,2635 \\
\hline
\end{tabular}

LEGENDA: $\mathrm{VI}=$ valor de importância; $\mathrm{DA}=$ densidade absoluta; $\mathrm{DR}=$ densidade relativa; $\mathrm{FA}=$ frequência absoluta; FR = frequência relativa; $D o A=$ dominância absoluta; DoR = dominância relativa.

Com a exclusão dos indivíduos de oiti é possível visualizar e analisar melhor a importância das demais espécies para a arborização das ruas de Bonito-MS com base na densidade, porte e distribuição espacial destas, pois o domínio de L. tomentosa na composição acarreta em valores exacerbados dos parâmetros fitossociológicos e mascara a real significância e expressividade de cada uma delas.

Embora as árvores de Caesalpinia pluviosa (sibipiruna) apresentem um alto valor de importância, não é recomendado o uso futuro, pois esta apresentou susceptibilidade a ataque de cupins comprometendo a performance desta espécie na arborização local. A susceptibilidade da espécie à cupins também foi verificada em Rio Claro, onde 37\% dos indivíduos amostrados estavam infestados por cupins (FERRETTI et al., 2011).

Como a área de copa foi a variável utilizada no cálculo da dominância, o índice de valor de importância destacou espécies que apresentaram maior área de copa ( $T$. catappa e Ficus sp, por exemplo), mesmo em detrimento de algumas espécies que apresentaram maior frequência absoluta. Isto indica que estas espécies estão contribuindo de forma mais significativa para a criação de um ambiente termicamente mais confortável através da sombra produzida pela copa das árvores.

T. catappa foi a espécie mais frequente no inventário realizado por Maria, Biondi e Bobrowski (2016) em Itanhaém-SP, e os autores afirmam que o plantio em larga escala dessa 
espécie no município se deve a grande atratividade da população pela sombra proporcionada. Lima Neto e Melo e Souza (2011) alertam para o uso de Ficus sp na arborização viária, afirmando que esta espécie possui crescimento agressivo de raízes superficiais, além de raízes adventícias, que danificam as calçadas, portanto deve ser restringida a locais com grande área permeável.

Se considerada a determinação do valor de importância (VI) com a substituição da densidade relativa pelo índice de performance (IPE) relativo por espécie (Tabela 3), tem-se a valorização das espécies $M$. exotica (murta), M. indica (mangueira), S. molle (aroeira-salsa), $T$. avellanedae (ipê-rosa) e $P$. guajava (goiabeira), com redução da importância das espécies ipê (não identificado), Bauhinia sp. (pata-de-vaca), P. aquatica (embiratanha), L. indica (extremosa) e T. roseoalba (ipê-branco). Ficus sp. (fícus) foi excluída do ranking, enquanto M. lepidopetalus (água-pomba) passou a compô-lo.

Tabela 3. As 15 espécies de maior valor de importância na arborização viária de Bonito-MS, utilizando o IPE na composição do VI

Table 3. The 15 species with higher importance value in the afforestation of Bonito-MS, using the IPE to calculate the VI

\begin{tabular}{lrrrrrr}
\hline \multicolumn{7}{c}{ ANÁLISE FITOSSOCIOLÓGICA } \\
\hline ESPÉCIE & VI & IPE & FA & FR & DoA & DoR \\
\hline Licania tomentosa (Benth.) Fritsch & 109,94 & 1,93 & 100,00 & 9,83 & 461114,69 & 98,1850 \\
Caesalpinia pluviosa DC. & 7,62 & 0,58 & 64,71 & 6,36 & 3196,20 & 0,6806 \\
Murraya exótica L. & 7,32 & 2,09 & 52,94 & 5,20 & 137,40 & 0,0293 \\
Bauhinia sp. & 7,07 & 1,75 & 52,94 & 5,20 & 569,61 & 0,1213 \\
Tabebuia avellanedae Lorentz ex Griseb. & 6,59 & 1,90 & 47,06 & 4,62 & 335,74 & 0,0715 \\
Mangifera indica L. & 6,45 & 1,77 & 47,06 & 4,62 & 282,23 & 0,0601 \\
Pachira aquática Aubl. & 5,90 & 1,73 & 41,18 & 4,05 & 559,58 & 0,1191 \\
Ipê (não identificado) & 5,65 & 1,72 & 35,29 & 3,47 & 2190,44 & 0,4664 \\
Schinus molle L. & 5,12 & 2,21 & 29,41 & 2,89 & 85,88 & 0,0183 \\
Tabebuia roseoalba (Ridl.) Sandwith & 4,91 & 1,99 & 29,41 & 2,89 & 137,40 & 0,0293 \\
Delonix regia (bojer ex hook.) raf & 4,69 & 1,77 & 29,41 & 2,89 & 133,46 & 0,0284 \\
Psidium guajava L. & 4,53 & 2,21 & 23,53 & 2,31 & 22,46 & 0,0048 \\
Malpighia emarginata DC. & 4,53 & 2,21 & 23,53 & 2,31 & 22,06 & 0,0047 \\
Lagerstroemia indica L. & 3,97 & 2,21 & 17,65 & 1,73 & 96,38 & 0,0205 \\
Melicoccus lepidopetalus Radlk. & 3,95 & 2,21 & 17,65 & 1,73 & 23,27 & 0,0050 \\
\hline
\end{tabular}

LEGENDA: VI = valor de importância; IPE = Índice de Performance por Espécie; FA= frequência absoluta; $\mathrm{FR}=$ frequência relativa; $\mathrm{DoA}=$ dominância absoluta; $\mathrm{DoR}=$ dominância relativa.

Segundo Bobrowski (2014), a substituição da densidade pelo IPE como um dos fatores descritivos do VI permite a reclassificação das espécies de duas maneiras: destaque das espécies com árvores em melhor condição, expressando melhor adaptação destas à arborização de ruas da cidade, e depreciação do VI das espécies que apresentaram mais árvores em condição inferior. Dessa maneira, o VI composto pelo IPE, ao depreciar as espécies em 
condições relativamente ruins, dá um panorama mais realista da importância das mesmas na composição da arborização de ruas, permitindo a tomada de ações de manutenção e manejo mais adequadas, bem como demonstrando espécies indicadas e promissoras para novos plantios.

Entretanto, neste estudo C. pluviosa (sibipiruna) foge a este padrão de reclassificação, pois apresentou um dos piores IPE $(0,58)$ entre todas as espécies e ainda assim ocupa a $2^{\text {a }}$ posição no ranking. Este fato se deve à área de copa total amostrada para a espécie $(14.018,54$ $\left.\mathrm{m}^{2}\right)$ ser superior às demais, aumentando de maneira significativa a dominância relativa e consequentemente, o valor de importância da espécie, mesmo apresentando diversos problemas de adaptabilidade e até mesmo oferecendo riscos nas ruas do município.

Os resultados apresentados na Figura 2 demonstram graficamente 0 valor da dominância relativa das espécies com melhores VI e permite compreender o peso desta variável na classificação da $C$. pluviosa (sibipiruna) como primeira espécie, desconsiderando $L$. tomentosa, de maior importância para a arborização de Bonito-MS.

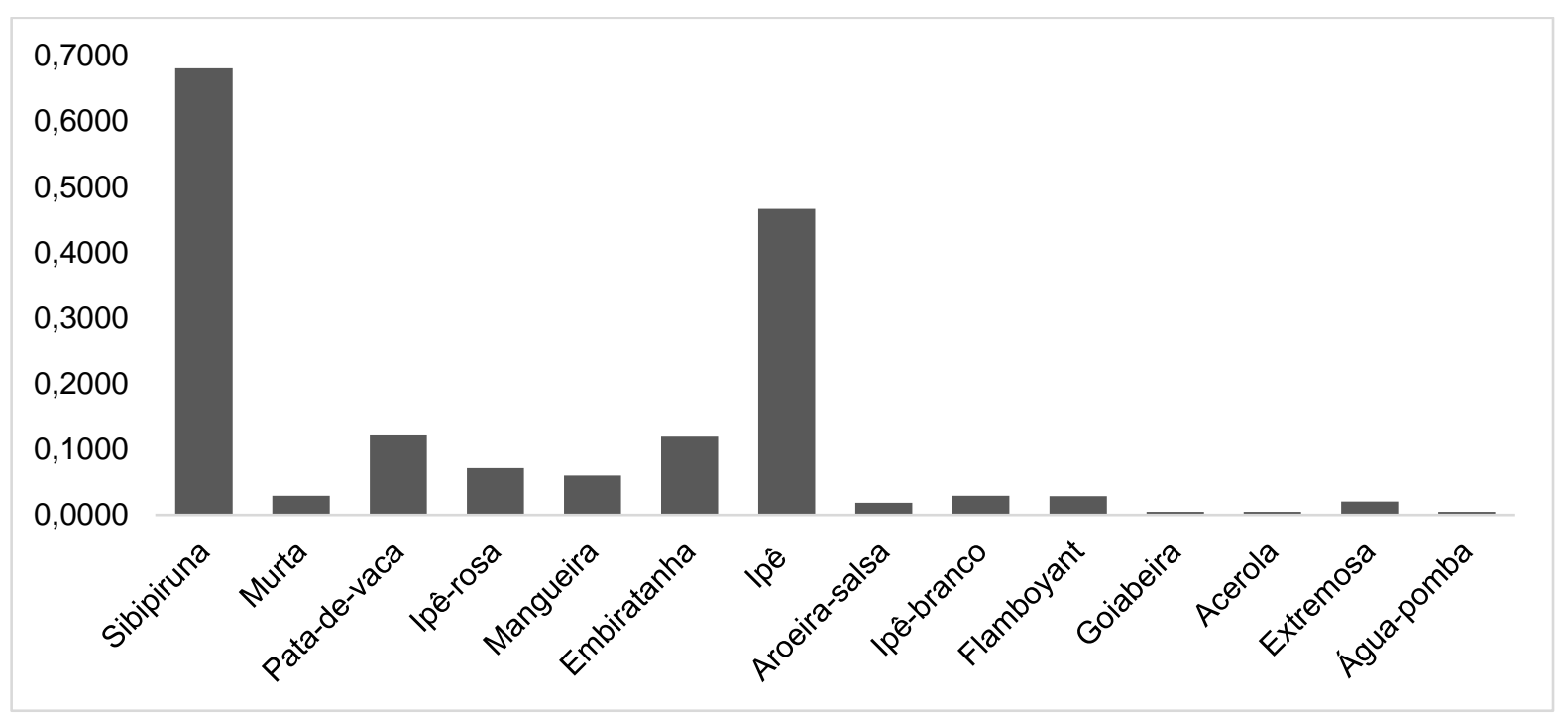

Figura 1. Dominância relativa das espécies com maiores VI em Bonito-MS

Figure 2. Relative dominance of the species with greater VI in Bonito-MS

Os indivíduos de ipê (não identificados) representam a segunda maior dominância $(0,4664)$, portanto, a segunda maior área de copa da arborização de Bonito-MS $\left(9.607,27 \mathrm{~m}^{2}\right)$, contribuindo positivamente para o conforto térmico da cidade. As espécies B. variegata (pata-devaca) e T. avellanedae (ipê-rosa), além de apresentarem valores altos de IPE (1,75 e 1,90, respectivamente), proporcionam bom sombreamento, por suas copas frondosas, constituindo, assim, alternativas potenciais para novos plantios no município. 
É interessante destacar o alto valor do IPE para L. tomentosa (oiti) com 1,93, indicando que apesar da homogeneização da arborização, esta é uma espécie bem adaptada às características locais.

Cabe apresentar que a espécie $H$. ochraceus (ipê-amarelo), que não aparece nas tabelas apresentadas anteriormente pois não ficou classificada entre as 15 primeiras posições em valor de importância, obteve valor de IPE 2,21, demonstrando que é uma espécie bem adaptada às condições urbanas do município e representando uma alternativa promissora para novos plantios. Handroanthus ochraceus, conhecida como ipê-amarelo, é uma árvore nativa, caducifólia, que pode atingir até $16 \mathrm{~m}$ de altura e ocorre no Cerrado e Mata Atlântica (ESPIRITO SANTO; SILVA-CASTRO; RAPINI, 2013). Segundo Moura e Corrêa (2015), H. ochraceus é uma espécie de grande importância nas áreas de vegetação urbana do cerrado, pois sua floração ocorre na estação seca do ano, diferentemente da maioria das outras espécies, portanto, desempenham papel fundamental na manutenção da fauna.

\section{CONCLUSÕES}

A partir dos resultados obtidos, verificou-se homogeneização da arborização viária do município de Bonito-MS com a predominância de Licania tomentosa (oiti). Embora a espécie apresente-se bem adaptada às condições locais, conforme seu valor do índice de performance, sugere-se a adoção de medidas de introdução de novas espécies a fim de diminuir a frequência de L. tomentosa, bem como a suspensão de novos plantios desta espécie, de modo a contribuir para a melhoria estética e ecológica da cidade e evitar a perda do patrimônio arbóreo do município em caso de surto de praga ou doença.

Espécies promissoras, de acordo com o índice de performance, que podem ser incrementadas em novos plantios de modo a alterar a proporção de oiti são: Lagerstroemia indica (extremosa), Bauhinia sp. (pata-de-vaca), Tabebuia roseoalba (ipê-branco) e Tabebuia avellanedae (ipê-rosa). Handroanthus ochraceus (ipê-amarelo), que não ficou classificada entre as espécies mais representativas da arborização de Bonito-MS também possui grande potencial para futuros plantios.

Os dados obtidos com os estudos fitossociológicos permitem incrementar a análise de dados obtidos pelo inventário, e, especialmente o IPE, auxilia a tomada de decisões para o manejo e futuros plantios da arborização viária. 


\section{AGRADECIMENTOS}

À Fundação Neotrópica do Brasil e à Prefeitura Municipal de Bonito (MS) pela oportunidade de realização deste trabalho e apoio financeiro.

\section{REFERÊNCIAS}

ARAÚJO, M. N.; ARAÚJO, A. J. Arborização Urbana. Curitiba: CREA, 2011.

BOBROWSKI, R. Gestão da arborização de ruas: ferramentas para o planejamento técnico e participativo. 178f. Tese (Doutorado em Engenharia Florestal) - Setor de Ciências Agrárias, Universidade Federal do Paraná, Curitiba, 2014.

BOBROWSKI, R. A floresta urbana e a arborização de ruas. In: BIONDI, D. Floresta urbana. Curitiba: A Autora, 2015, p. 81-108.

BOBROWSKI, R.; BIONDI, D. Distribuição e dinâmica da área de copa na arborização de ruas de Curitiba, Paraná, Brasil, no período de 1984-2010. Revista Árvore, Viçosa, v.36, n.4, p.625635, 2012.

ESPIRITO SANTO, F.S.E.; SILVA-CASTRO, M. M.; RAPINI, A. Flora da Bahia: Bignoniaceae 2 - Aliança Tabebuia. Sitientibus série Ciências Biológicas. v.13. 2013.

FEHLAUER, T. J.; RODRIGUER-OTUBO, B. M.; SANDRINI, M.; DESTRO, D. Caracterização da produção de genótipos de banana introduzidos na região de Bonito - MS. Revista Brasileira de Fruticultura, Cruz das Almas, v. 32, p. 938-943, 2010.

FERREIRA, F. A.; GASPAROTTO, L.; LIMA, M. I. P. M. Uma ferrugem, causada por Phakopsora tomentosae em oiti, em Manaus. Fitopatologia Brasileira, Brasília, v.26, n.2, p. 206 - 208, 2001.

FERRETTI, A. B. S. M.; NASCIMENTO, J. P. R.; BUENO, O. C.; CHAUD, V. C. T.; Monitoramento de cupins subterrâneos na arborização urbana de Rio Claro - SP. Revista Ciência em extensão, Rio Claro, v.7, n.3, p.44, 2011.

GIEHL, E. L. H.; BUDKE, J. C. Aplicação do método científico em estudos fitossociológicos no Brasil: em busca de um paradigma. In: FELFILI, J. M.; EISENLOHR, P. V.; MELO, M. M. R. F.; ANDRADE, L. A.; MEIRA NETO, J. A. A. Fitossociologia no Brasil: métodos e estudos de casos. Viçosa: Editora UFV, 2011. p. 23-43.

GONÇALVES, A. O.; PEREIRA, N. R; da COSTA, L. L. Caracterização climática e aptidão das culturas anuais e perenes no zoneamento pedoclimático do Estado do Mato Grosso do Sul - 1ª fase. Dados eletrônicos - Rio de Janeiro: Embrapa Solos, 2006.

LARA, J. S.; ALVES, E. D. L.; CARNEIRO, F. M. Diagnóstico da composição arbórea da cidade de Israelândia-GO, Brasil. RevSBAU, Piracicaba, v.9, n.2, p. 134-147, 2014.

LIMA NETO, E. M. Índices e métricas para a gestão das árvores de ruas de Boa Vista-RR a partir de cadastro espacial. 169f. Tese (Doutorado em Engenharia Florestal) - Setor de Ciências Agrárias, Universidade Federal do Paraná, Curitiba, 2014. 
LIMA NETO, E. M.; MELO E SOUZA, R. Comportamento e características das espécies arbóreas nas áreas verdes públicas de Aracaju, Sergipe. Scientia Plena, Aracaju, v. 7, p. 1-10, 2011.

LORENZI, H. Árvores brasileiras: manual de identificação e cultivo de plantas arbóreas nativas do Brasil. 5.ed. Nova Odessa: Editora Plantarum, 2008.

MARIA, T. R. B. C.; BIONDI, D., BOBROWSKI, R. Inventário florístico quali-quantitativo das vias púbicas de Itanhaém -SP. RevSBAU, Piracicaba, v.11, n.4, p. 79-97, 2016.

MILANO, M S. Avaliação e análise da arborização de ruas de Curitiba-PR. 130 f. Dissertação (Mestrado em Ciências Florestais) - Setor de Ciências Agrárias, Universidade Federal do Paraná, Curitiba, 1984.

MORO, M. F.; MARTINS, F. R. Métodos de levantamento do componente arbóreo-arbustivo. In: FELFILI, J.M.; EISENLOHR, P. V.; MELO, M. M. R. F.; ANDRADE, L. A.; MEIRA NETO, J. A.

A. Fitossociologia no Brasil: métodos e estudos de casos. Viçosa: Editora UFV, 2011. p. 174212.

MOURA, A. S.; CORRÊA, B.S. Ornitofilia em Handroanthus ochraceus (Cham.) Mattos (Bignoniaceae) em um fragmento de floresta decidual do norte de Minas Gerais. Regnellea Scientia. Poços de Caldas, v.2, n.1, p. 23-39. 2015.

NIELSEN, A.B.; ÖSTBERG, J.; DELSHAMMAR, T. Review of urban tree inventory methods used to collect data at single-tree level. Arboriculture \& Urban Forestry, Champaign, v.40, n.2, p.96-111, 2014.

RESTREPO, L. A. V. Paisajismo y ecología del paisaje en la gestión de la arborización de calles. Una referencia a la ciudad de Medellín, Colombia. Gestión y Ambiente, Bogotá, v.10, n.4, p. 131-140, 2007.

SANTOS, A. F.; JOSÉ, A. C.; SOUSA, P. A. Fitossociologia e diversidade de espécies arbóreas das praças centrais do município de Gurupi-TO. RevSBAU. Piracicaba, v.8, n.4, p. 36-46, 2013.

SILVA, A. G.; CARDOSO, A. L.; RAPHAEL, M. Diagnóstico quali-quantitativo da arborização viária da cidade de Jerônimo Monteiro-ES. Enciclopédia Biosfera, Goiânia, v.8, n.14, p.11791188, 2012.

SILVA, A. G.; GONÇALVES, W. Inventário e diagnóstico da arborização da cidade de CajuriMG. Enciclopédia Biosfera, Goiânia, v.8, n.15, p.1102-1113, 2012.

ZAMPRONI, K.; BIONDI, D.; MARIA, T. R. B. C.; LOUVEIRA, F. A. Diagnóstico qualiquantitativo da arborização viária de Bonito, Mato Grosso do Sul. Revista Floresta, Curitiba, v. 48, n. 2, p. 235-244, 2018. 